\title{
MOLECULAR IDENTIFICATION OF TRICHODERMA STRAINS COLLECTED TO DEVELOP PLANT GROWTH-PROMOTING AND BIOCONTROL AGENTS
}

\author{
Michał OSKIERA*1, Magdalena SZCZECH ${ }^{1}$, Grzegorz BARTOSZEWSKI ${ }^{2}$ \\ ${ }^{1}$ Department of Microbiology, Research Institute of Horticulture, 96-100 Skierniewice, Poland \\ ${ }^{2}$ Department of Plant Genetics Breeding and Biotechnology, Warsaw University of Life Sciences \\ (SGGW), Nowoursynowska 159, 02-776 Warsaw, Poland
}

Received: May, 2015; Accepted: June, 2015

\begin{abstract}
Trichoderma strains that are beneficial to both the growth and health of plants can be used as plant growth-promoting fungi (PGPF) or biological control agents (BCA) in agricultural and horticultural practices. In order to select PGPF or BCA strains, their biological properties and taxonomy must be carefully studied. In this study, 104 strains of Trichoderma collected at geographically different locations in Poland for selection as PGPF or BCA were identified by DNA barcoding, based on the sequences of internal transcribed spacers 1 and 2 (ITS1 and 2) of the ribosomal RNA gene cluster and on the sequences of translation elongation factor 1 alpha (tef1), chitinase 18-5 (chi18-5), and RNA polymerase II subunit ( $r p b 2)$ gene fragments. Most of the strains were classified as: T. atroviride (38\%), T. harzianum (21\%), T. lentiforme (9\%), T. virens (9\%), and T. simmonsii (6\%). Single strains belonging to T. atrobrunneum, T. citrinoviride, T. crassum, $T$. gamsii, $T$. hamatum, $T$. spirale, $T$. tomentosum, and $T$. viridescens were identified. Three strains that are potentially pathogenic to cultivated mushrooms belonging to T. pleuroticola and T. aggressivum f. europaeum were also identified. Four strains: TRS4, TRS29, TRS33, and TRS73 were classified to Trichoderma spp. and molecular identification was inconclusive at the species level. Phylogeny analysis showed that three of these strains TRS4, TRS29, and TRS33 belong to Trichoderma species that is not yet taxonomically established and strain TRS73 belongs to the $T$. harzianum complex, however, the species could not be identified with certainty.
\end{abstract}

Key words: fungal barcoding, multilocus sequence analysis, phylogeny, species identification

\section{INTRODUCTION}

Trichoderma fungi occur worldwide and are present in different geographic regions and climatic zones (Hoyos-Carvajal et al. 2009). Trichoderma can be isolated from the air, water reservoirs, soils, plants, animals, and other fungi. Several species reveal high association with plants and are often isolated from different soils as the predominant species in the plant root ecosystem (Kredics et al. 2014). Trichoderma plays a significant role as a decaying wood decomposer and sometimes occurs as a plant endophyte (Gazis \& Chaverri 2010; Chaverri et al. 2011). First interest in Trichoderma was related to its cellulolytic properties. Then, it was found that the fungi of this genus may produce multiple enzymes that can be of great importance in several industries and can be used in agricultural practice as plant growth-promoting fungi (PGPF) or biocontrol agents (BCA) to control fungal diseases or nematodes (Hermosa et al. 2013; Stewart \& Hill 2014; Monfil \& Casas-Flores 2014). Plant pathogens can be limited due to the facility of mycoparasitism, antibiosis, and competition of nutrients and space with Trichoderma. There are also other ways in which Trichoderma may benefit plant cultivation, such as enhancing root system and plant development, inducing resistance, and increasing the availability of nutrients (Benítez et al. 2004; Harman et al. 2004; Harman 2006). 
Commercial products based on Trichoderma are available on the market and are used to protect plants or to stimulate plant growth. These products are based on strains belonging to the following species: T. asperellum (T-203), T. atroviride (SC1, P1, or IMI206040), T. harzianum (NF-9, T1, T22, or 2413), and T. virens (G-6, G-6-5, or G-11) (Hermosa et al. 2000; Kullnig et al. 2001; Dodd et al. 2003; Longa et al. 2009; Chaverri et al. 2015). However, since the application of beneficial microbes has become an integrated component of plant management in agricultural or horticultural practice, interest in developing new products based on Trichoderma is growing. Studies conducted at the Research Institute of Horticulture in Skierniewice are aimed at developing Trichoderma preparations with the use of agricultural wastes to be applied in the production of vegetables and other crops for plant growth promotion, protection, and enhanced yield quality (Kancelista et al. 2013; Smolińska et al. 2014a, b). The first step in such studies should be species identification of promising Trichoderma strains. Correct species identification is necessary due to Trichoderma similarity in morphology and complex taxonomy (Hermosa et al. 2000). Especially Trichoderma strains that are potentially dangerous to cultivated mushrooms (T. aggressivum f. europaeum, T. aggressivum f. aggressivum, $T$. pleurotum, and $T$. pleuroticola) should be excluded from PGPF or BCA strain selection (Komoń-Żelazowska et al. 2007). Morphology alone is insufficient in accurate Trichoderma species identification, particularly when such a group of species as the T. harzianum complex or T. viridescens complex are investigated, as they are genetically diverse and characterized by variable morphology (Chaverri et al. 2003; Druzhinina et al. 2010; Jaklitsch et al. 2013). Recently, Chaverri et al. (2015) re-evaluated the taxonomy of $T$. harzianum; they distinguished 14 species within the $T$. harzianum complex and reported that none of the T. harzianum BCA strains belong to T. harzianum sensu stricto.

DNA-based methods used to build the modern taxonomy of the Trichoderma genus and DNA barcoding are now routinely used in species identification. Accurate Trichoderma species discrimination can be done based on DNA sequence analysis of internal transcribed spacers (ITS) 1 and 2 of the rDNA gene cluster and fragments of the tef1, rpb2, chi18-5, and actin (act) or calmodulin (cal1) genes (Kindermann et al. 1998; Dodd et al. 2003; Druzhinina et al. 2005, 2010; Jaklitsch et al. 2006; Samuels et al. 2006; Gal-Hemed et al. 2011; Błaszczyk et al. 2011; Atanasova et al. 2013; Jaklitsch \& Voglmayr 2015). The taxonomy of Trichoderma is continuously adjusted and updated (Jaklitsch 2011; Atanasova et al. 2013; Jaklitsch \& Voglmayr 2015; Chaverri et al. 2015). More and more strains are isolated from different geographic regions (Mulaw et al. 2010; GalHemed et al. 2011) and novel DNA sequences are available, hence still more Trichoderma species and clades are being established. Recently, 228 species of Trichoderma and several not yet taxonomically characterized species were distinguished (Jaklitsch \& Voglmayr 2015).

The aim of this study was to conduct the molecular identification of Trichoderma strains collected in order to select PGPF or BCA strains suitable for application in agricultural and horticultural production.

\section{MATERIALS AND METHODS}

\section{Trichoderma strains}

A total of 104 Trichoderma strains (Table 1) collected for the selection of PGPF or BCA strains were subjected to species identification. Based on colony morphology and microscopic observations, the strains were preliminarily recognized as T. atroviride, T. harzianum, and T. virens (Szczech et al., unpublished). $T$. atroviride strains were previously initially identified based on ITS1 and 2, and tef 1 sequences (Skoneczny et al. 2015). The reference strains were as follows: T. atroviride IMI 206040 (CABI Europe, UK), T. afroharzianum T22 (ATCC 20847, LGC Standards, Poland), T. virens Gv29-8 (FGSC 10586, Fungal Genetics Stock Center, School of Biological Sciences, University of Missouri, Kansas City, USA), and T. aggressivum f. europaeum (CBS100526T, Centraalbureau voor Schimmelcultures, the Netherlands). All strains were preserved in $-80{ }^{\circ} \mathrm{C}$ on PDA discs submerged in glycerol. 


\section{DNA isolation}

Fungal liquid cultures were initiated in Potato Dextrose Broth (Fluka, Buchs, Switzerland) and maintained on a rotary shaker at $25{ }^{\circ} \mathrm{C}$ for one week. Fungal cells were collected by centrifugation and homogenized in liquid nitrogen with quartz sand (Sigma-Aldrich, St. Louis, MO, USA). DNA was isolated using the $2 \times$ CTAB method (Aldrich $\&$ Cullis 1993) and further purified using the DNeasy Plant Mini Kit (Qiagen, Hilden, Germany) according to the manufacturer's instructions, with minor modifications. Polyvinylpyrrolidone (PVP) and polyvinylpolypyrrolidone (PVPP) were used according to Vandroemme et al. (2008), and proteinase $\mathrm{K}$ and RNase A were added to the lysis buffer. Both the purity and quantity of DNA were checked by agarose gel electrophoresis, determined with the NanoDrop 8000 spectrophotometer (Thermo Scientific, Waltham, MA, USA), and the DNA concentration was adjusted to $10 \mathrm{ng} \cdot \mu \mathrm{l}^{-1}$.

\section{PCR and sequencing}

PCR was used to amplify the internal transcribed spacer regions 1 and 2 (ITS1 and 2) of the rRNA gene cluster and the translation elongation factor 1-alpha (tef1), chitinase 18-5 (chi18-5), and RNA polymerase II subunit ( $r p b 2)$ gene fragments. The PCR mixture $(20 \mu \mathrm{l})$ contained $1 \times$ PCR buffer, $1.5 \mathrm{mM} \mathrm{MgCl} 2,0.25 \mathrm{mM}$ dNTPs, $0.5 \mu \mathrm{M}$ of each primer, $4 \mu \mathrm{g}$ bovine serum albumin (BSA), $1 \mathrm{U}$ of DreamTaq Polymerase (Fermentas, Vilnius, Lithuania), and $20 \mathrm{ng}$ of DNA template. The primers used in this study are listed in Table 2. The ITS region and chi18-5 gene fragment were amplified using the following cycling parameters: $3 \mathrm{~min}$ at $94{ }^{\circ} \mathrm{C}$ followed by 35 cycles each of $30 \mathrm{~s}$ at $94{ }^{\circ} \mathrm{C}, 30 \mathrm{~s}$ at $55^{\circ} \mathrm{C}, 1 \mathrm{~min}$ at $72{ }^{\circ} \mathrm{C}$ and, finally, $10 \mathrm{~min}$ at $72{ }^{\circ} \mathrm{C}$. For amplification of the $t e f 1$ gene fragment, the following program was used: $1 \mathrm{~min}$ at $94^{\circ} \mathrm{C}$, followed by 35 cycles each of $1 \mathrm{~min}$ at $94^{\circ} \mathrm{C}, 1 \mathrm{~min}$ at $59^{\circ} \mathrm{C}$, $50 \mathrm{~s}$ at $74{ }^{\circ} \mathrm{C}$ and, finally, $7 \mathrm{~min}$ at $74{ }^{\circ} \mathrm{C}$ (www.isth.info). For the $r p b 2$ gene fragment the following protocol was used: 3 min at $94{ }^{\circ} \mathrm{C}, 5$ cycles each of $45 \mathrm{~s}$ at $94{ }^{\circ} \mathrm{C}, 45 \mathrm{~s}$ at $60{ }^{\circ} \mathrm{C}$, and $2 \mathrm{~min}$ at $72{ }^{\circ} \mathrm{C}$, followed by 5 cycles with the temperature decreasing by $1.0^{\circ} \mathrm{C}$ per cycle from $58^{\circ} \mathrm{C}$ to $54^{\circ} \mathrm{C}$, followed by 30 cycles at $54^{\circ} \mathrm{C}$, and, finally, $10 \mathrm{~min}$ at $72{ }^{\circ} \mathrm{C}$ (www.isth.info). To confirm DNA amplification, $1 \mu 1$ of each PCR reaction was electrophoresed with $1.5 \%$ agarose $-0.5 \times$ Tris-borateEDTA (TBE) gels containing ethidium bromide and visualized under UV light using the GelDoc-It System with VisionWorks LS 6.7.4 (UVP, Upland, CA, USA). Prior to sequencing, enzymatic ExoSAP clean-up was performed according to the manufacturer's instructions (Affymetrix, Santa Clara, CA, USA). PCR products were diluted with $\mathrm{H}_{2} \mathrm{O}$ and mixed with 5 pmol of primer up to $6 \mu 1$, and sequenced by Genomed S.A. (Warsaw, Poland) or the DNA Sequencing and Oligonucleotide Synthesis Lab (IBB PAS, Warsaw, Poland). All amplicons were sequenced from both directions with primers used for amplification (Table 2). Additional sequencing primer tef790 was used for sequencing tef 1 of the T. harzianum strains (Table 2). Amplification and sequencing of chi18-5 for three strains (TRS4, TRS29, and TRS33) was done with ech1f and ech1r primers (Table 2). Sequences of the collected strains are available at the National Center for Biotechnology Information (NCBI) GenBank (accession numbers are provided in Table 1).

\section{Bioinformatic analysis and Trichoderma species identification}

The raw sequence reads of ITS1 and ITS2, tef1, rpb2, and chi18-5 were checked for quality, trimmed, manually edited and assembled using CLC Genomics Workbench 7.5 (CLCBio, Aarhus, Denmark). Taxonomic identification based on ITS 1 and 2 was performed with TrichOKEY2 (isth.info/tools/molkey/index.php, Druzhinina et al. 2005). Sequences of ITS 1 and 2, tef 1 , and $r p b 2$ were also analysed using TrichoBLAST (isth.info/tools/blast/blast.php; Kopchinskiy et al. 2005). To conduct taxonomic identification, intron 4 of the tef 1 gene was selected with TrichoMARK (isth.info/tools/blast/preblast.php) and compared with the International Subcommission on Trichoderma and Hypocrea Taxonomy (ISTH) database (www.isth.info) using TrichoBLAST, and also with publicly available sequences deposited at NCBI (www.ncbi.nlm.nih.gov) by using a basic local alignment search tool (BLAST) (Altschul et al. 1997). 
Table 1. Origin of the Trichoderma strains used in this study and sequences from NCBI GenBank accession numbers (species order according to general Trichoderma taxonomy)

\begin{tabular}{|c|c|c|c|c|c|c|}
\hline \multirow{2}{*}{ Strain no. } & \multirow{2}{*}{ Source } & \multirow{2}{*}{ Origin in Poland } & \multicolumn{4}{|c|}{ NCBI GenBank accession number } \\
\hline & & & ITS1 and 2 & tef 1 & chi18-5 & $r p b 2$ \\
\hline \multicolumn{7}{|c|}{ T. atroviride Bissett $^{\mathrm{a}}$} \\
\hline TRS1 & mushroom compost & Maków & KJ786739 & KJ786820 & KP009492 & KP009036 \\
\hline TRS2 & dust, mushroom farm & composting factory, Łódź & KJ786749 & KJ786830 & KP009508 & KP009052 \\
\hline TRS3 & mushroom farm, floor & Maków & KJ786743 & KJ786824 & KP009507 & KP009051 \\
\hline TRS5 & mushroom compost & composting factory, Łódź & KJ786741 & KJ786822 & KP009502 & KP009046 \\
\hline TRS6 & mushroom compost & Sława & KJ786750 & KJ786831 & KP009509 & KP009053 \\
\hline TRS7 & mushroom farm & Bieruń & KJ786747 & KJ786828 & КР009472 & КР009015 \\
\hline TRS9 & mushroom farm, air & Maków & KJ786738 & KJ786819 & KP009506 & KP009050 \\
\hline TRS10 & not known & Turek & KJ786754 & KJ786835 & KP009515 & KP009057 \\
\hline TRS11 & not known & $\mathrm{WU}^{\mathrm{b}}$ & KJ786735 & KJ786816 & KР009483 & KP009027 \\
\hline TRS12 & not known & $\mathrm{WU}^{\mathrm{b}}$ & KJ786737 & KJ786818 & KР009485 & KP009029 \\
\hline TRS13 & not known & $W^{b}$ & KJ786736 & KJ786817 & KP009484 & KP009028 \\
\hline TRS14 & mushroom compost & $\mathrm{LMC}^{\mathrm{c}}$ & KJ786725 & KJ786806 & KP009486 & KP009030 \\
\hline TRS15 & forest wood & Głuchów & KJ786724 & KJ786805 & KP009499 & KP009043 \\
\hline TRS16 & not known & not known & KJ786753 & KJ786834 & KР009512 & KP009056 \\
\hline TRS17 & mushroom compost & Skierniewice & KJ786734 & KJ786815 & KP009482 & KP009026 \\
\hline TRS18 & mushroom compost & Maków & KJ786757 & KJ786839 & КР009519 & KP009061 \\
\hline TRS19 & soil & not known & KJ786732 & KJ786813 & КР009480 & KP009024 \\
\hline TRS20 & mushroom farm, floor & Sierakowice Lewe & KJ786730 & KJ786811 & КР009489 & KP009033 \\
\hline TRS21 & soil & Topolowo near Sochacz & KJ786727 & KJ786808 & KP009487 & KP009031 \\
\hline TRS22 & soil & Wiskitki & KJ786726 & KJ786807 & KР009491 & KP009035 \\
\hline TRS24 & mushroom farm, wall & Ignanie Nowe & KJ786722 & KJ786803 & КР009498 & КР009042 \\
\hline TRS25 & mushroom farm, wall & Ignanie Nowe & KJ786731 & KJ786812 & КР009490 & KP009034 \\
\hline TRS26 & soil & Lipniak near Kock & KJ786751 & KJ786832 & КР009510 & KP009054 \\
\hline TRS28 & mushroom farm, floor & Kolonia Bolimowska & KJ786721 & KJ786802 & KР009494 & KP009038 \\
\hline TRS30 & soil & Skierniewice & KJ786720 & KJ786801 & КР009493 & KР009037 \\
\hline TRS31 & soil & Balcerów & KJ786740 & KJ786821 & КР009501 & KP009045 \\
\hline TRS32 & mushroom farm, air & Maków & KJ786744 & KJ786825 & КР009504 & KP009048 \\
\hline TRS34 & not known & not known & KJ786719 & KJ786800 & KР009497 & KP009041 \\
\hline TRS36 & soil & Katne & KJ786729 & KJ786810 & KР009488 & KP009032 \\
\hline TRS38 & soil & Myślibórz & KJ786733 & KJ786814 & KР009481 & KP009025 \\
\hline TRS39 & soil & Myślibórz & KJ786728 & KJ786809 & КР009479 & KP009023 \\
\hline TRS40 & organic manure & ecological farm, Rzeczyce & KJ786755 & KJ786836 & KP009516 & KP009058 \\
\hline TRS42 & soil & Radzanów & KJ786752 & KJ786833 & КР009511 & КР009055 \\
\hline TRS43 & forest soil & Wielkopolski National Park ${ }^{\mathrm{d}}$ & KJ786748 & KJ786829 & 9473 & KP009016 \\
\hline TRS44 & not known & not known & KJ786756 & KJ786837 & KP0 & КР009059 \\
\hline TRS45 & mushroom compost & Maków & KJ786718 & KJ786799 & KP009496 & KP009040 \\
\hline TRS46 & not known & not known & KJ786723 & KJ786804 & KP009495 & КР009039 \\
\hline TRS47 & not known & not known & KJ786746 & KJ786827 & KP009503 & KP009047 \\
\hline TRS68 & mushroom compost & Skierniewice & KJ786745 & KJ786826 & KP009505 & KP009049 \\
\hline \multicolumn{7}{|c|}{ T. gamsii Samuels \& Druzhinina } \\
\hline TRS123 & soil & Strobów & KP009330 & KP008922 & KP009528 & KP009065 \\
\hline TRS125 & soil & Wola Skromowska near Kock & KP009326 & KP008919 & KP009524 & KP009062 \\
\hline \multicolumn{7}{|c|}{ T. viridescens (A.S. Horne \& H.S. Williamson) Jaklitsch \& Samuels } \\
\hline TRS35 & soil & Lękosz near Brodnica & KP009338 & KP008930 & KP009522 & KP009074 \\
\hline \multicolumn{7}{|c|}{$T$. sp. $=T$. sp. 435 (Hoyos-Carvajal et al. 2009) and T. sp. C.P.K. 3606 (López-Quintero et al. 2013) } \\
\hline TRS4 & mushroom farm & Kolonia Bolimowska & KP009339 & KP008948 & KP009542 & KP009082 \\
\hline TRS29 & mushroom farm, wall & Maków & KP009340 & KP008949 & KP009543 & KP009083 \\
\hline TRS33 & mushroom farm, floor & Skierniewice & КР009341 & KP008950 & КР009544 & КР009084 \\
\hline \multicolumn{7}{|c|}{ T. hamatum (Bonorden) Bainier } \\
\hline TRS121 & forest wood & Głuchów & KP009342 & KP008953 & KP009537 & KP009085 \\
\hline TRS127 & not known & not known & KP009343 & KP008954 & KP009538 & KP009086 \\
\hline \multicolumn{7}{|c|}{ T. virens (J.H. Miller, Giddens \& A.A. Foster) Arx } \\
\hline TRS106 & mushroom compost & $\mathrm{LMC}^{\mathrm{c}}$ & KP009291 & KP008854 & KP009462 & KP009093 \\
\hline TRS107 & mushroom compost & $\mathrm{LMC}^{\mathrm{c}}$ & КР009297 & KP008861 & KP009469 & KP009099 \\
\hline TRS108 & mushroom compost & $\mathrm{LMC}^{\mathrm{c}}$ & KР009290 & KP008857 & KP009465 & KР009092 \\
\hline TRS109 & mushroom compost & Skierniewice & КР009299 & KР008864 & КР009463 & KP009101 \\
\hline TRS110 & mushroom compost & Skierniewice & KP009298 & KP008863 & KP009461 & KP009100 \\
\hline TRS112 & mushroom compost & Skierniewice & KP009296 & KP008860 & KP009468 & KP009098 \\
\hline TRS114 & soil & Wola Skromowska near Kock & КР009293 & KP008862 & КР009467 & КР009095 \\
\hline TRS116 & soil & Radzanów & КР009289 & КР008856 & КР009470 & КР009091 \\
\hline TRS117 & not known & not known & КР009294 & KP008858 & КР009466 & КР009096 \\
\hline
\end{tabular}




\begin{tabular}{|c|c|c|c|c|c|c|}
\hline \multicolumn{7}{|c|}{ T. crassum Bissett } \\
\hline TRS113 & soil & Wola Skromowska near Kock & KP009300 & KP008865 & KP009380 & KP009102 \\
\hline \multicolumn{7}{|c|}{ T. harzianum Rifai (T. harzianum sensu stricto) } \\
\hline TRS53 & mushroom compost & Maków & KP009240 & KP008808 & KP009389 & KP009110 \\
\hline TRS54 & soil & not known & KP009217 & KP008833 & KP009408 & KP009129 \\
\hline TRS55 & mushroom farm & Maków & KP009211 & KP008803 & KP009400 & KP009121 \\
\hline TRS56 & mushroom compost & Częstochowa & KP009242 & KP008810 & KP009391 & KP009112 \\
\hline TRS57 & soil & Skierniewice & KP009241 & KP008809 & KP009390 & KP009111 \\
\hline TRS58 & soil & Wadowice & KP009214 & KP008818 & KP009403 & KP009124 \\
\hline TRS59 & soil & Balcerów & KР009237 & KP008790 & KP009386 & KP009107 \\
\hline TRS61 & mushroom compost & $\mathrm{LMC}^{\mathrm{c}}$ & KP009212 & KP008804 & KP009401 & KP009122 \\
\hline TRS62 & mushroom compost & Maków & КР009213 & КР008817 & KР009402 & KP009123 \\
\hline TRS65 & not known & Sokołów Podlaski & KP009247 & KP008799 & KP009396 & KP009117 \\
\hline TRS69 & mushroom farm & Maków & KP009216 & KP008821 & KP009406 & KP009127 \\
\hline TRS71 & soil & Janówek & KP009238 & KP008806 & KP009387 & KP009108 \\
\hline TRS72 & mushroom farm & Skierniewice & KP009236 & KP008789 & KP009385 & KP009106 \\
\hline TRS83 & soil & Nowy Białynin & КР009253 & KP008820 & KP009405 & KP009126 \\
\hline TRS87 & organic manure & ecological farm, Rzeczyce & КР009235 & КР008812 & КР009384 & KP009105 \\
\hline TRS88 & organic manure & ecological farm, Rzeczyce & КР009244 & KP008796 & КР009393 & KP009114 \\
\hline TRS89 & organic manure & ecological farm, Rzeczyce & КР009234 & КР008813 & KPOC & KP009104 \\
\hline TRS92 & soil & Radzanów & KР009243 & KP008811 & KР009392 & KP009113 \\
\hline TRS93 & soil & Radzanów & KP009246 & KP008798 & KP009395 & KP009116 \\
\hline TRS94 & soil & Lipniak near Kock & KP009250 & KP008802 & KР009399 & KP009120 \\
\hline TRS95 & soil & Janowo & КР009249 & KP008801 & KP009398 & KP009119 \\
\hline TRS122 & mushroom compost & Skierniewice & KP009239 & KP008807 & KP009388 & KP009109 \\
\hline \multicolumn{7}{|c|}{ T. atrobrunneum F.B. Rocha, P. Chaverri \& W. Jaklitsch, sp. nov. } \\
\hline TRS60 & mushroom compost & Balcerów & KP009260 & KP008874 & KP009432 & KP009143 \\
\hline TRS86 & soil & Rzeczyce & KP009254 & KP008876 & KP009431 & KP009142 \\
\hline TRS91 & soil & Wysoka & KP009261 & KP008875 & KP009438 & KP009144 \\
\hline \multicolumn{7}{|c|}{ T. lentiforme (Rehm) P. Chaverri, Samuels \& F.B. Rocha, comb. nov. } \\
\hline TRS35x & soil & Lękosz near Brodnica & KP009231 & KP008899 & KP009436 & KP009154 \\
\hline TRS58x & soil & Wadowice & KP009256 & KP008901 & KP009427 & KP009157 \\
\hline TRS63x & soil & production field, Skierniewice & КР009230 & КР008898 & KP009435 & KP009153 \\
\hline TRS64 & not known & Pszczyna & КР009229 & KP008897 & КР009434 & KP009152 \\
\hline TRS65x & not known & Sol & $\mathrm{KPO}$ & КР008896 & KP0 & KP009151 \\
\hline TRS74 & soil & Kątne & KP009258 & KP008904 & KP009429 & КР009159 \\
\hline TRS76 & soil & not known & KP009257 & KP008903 & KP009428 & KP009158 \\
\hline TRS78 & mushroom compost & Skórzec & КР009259 & KP008905 & KР009430 & КР009160 \\
\hline TRS79 & mushroom farm & Ignanie Nowe & KP009232 & KP008902 & KP009437 & KP009155 \\
\hline \multicolumn{7}{|c|}{ T. simmonsii P. Chaverri, F.B. Rocha, Samuels, Degenkolb \& W. Jaklitsch, sp. nov. } \\
\hline TRS66 & unknown & Parczew & KP009222 & KP008867 & KP009447 & KP009135 \\
\hline TRS67 & unknow & Radzyń Podlaski & KP009221 & KP008866 & KP009443 & KP009134 \\
\hline TRS75 & mushroom farm & Sierakowice Lewe & KP009223 & KP008871 & KP009448 & KP009136 \\
\hline TRS77 & mushroom farm, floor & Maków & КР009226 & КР008869 & КР009444 & KP009139 \\
\hline TRS80 & compost & Skie & КР009225 & КР008868 & КР009445 & KP009138 \\
\hline TRS85MO & mushroom farm, air & Sierakowice Lewe & KР009224 & KP008872 & KP009446 & KP009137 \\
\hline \multicolumn{7}{|c|}{ T. cf. harzianum } \\
\hline TRS73 & mushroom farm & Kolonia Bolimowska & KP009263 & KP008873 & KP009442 & KP009141 \\
\hline \multicolumn{7}{|c|}{ T. aggressivum f. europaeum Samuels \& W. Gams } \\
\hline TRS27 & mushroom farm, air & Sierakowice Lewe & KP009302 & KP008994 & KP009440 & KP009163 \\
\hline \multicolumn{7}{|c|}{ T. pleuroticola S.H. Yu \& M.S. Park } \\
\hline TRS70 & forest wood & Głuchów & KP009264 & KP008951 & KP009450 & KP009172 \\
\hline TRS120 & forest wood & Głuchów & KP009265 & KP008952 & KP009451 & KP009173 \\
\hline \multicolumn{7}{|c|}{ T. tomentosum Bissett } \\
\hline TRS82 & soil & Nowy Dwór & KP009287 & KP008914 & KP009455 & KP009177 \\
\hline \multicolumn{7}{|c|}{ T. spirale Bissett } \\
\hline TRS111 & soil & Rudzieniec & KP009301 & KP008963 & KP009471 & KP009182 \\
\hline \multicolumn{7}{|c|}{ T. citrinoviride Bissett } \\
\hline TRS119 & not known & $\mathrm{WU}^{\mathrm{b}}$ & KP009357 & KP008895 & KP009545 & KP009183 \\
\hline
\end{tabular}

${ }^{\mathrm{a}} T$. atroviride strains identification is in agreement with the identification presented by Skoneczny et al. (2015), which included ITS and tef 1 sequences listed above

${ }^{\mathrm{b}}$ provided by Wrocław University of Environmental and Life Sciences, Poland

${ }^{c}$ provided by the Laboratory of Mushroom Cultivars, Research Institute of Horticulture, Skierniewice, Poland

d provided by the Poznań University of Life Sciences, Poland 
Table 2. Primer names and sequences used for PCR amplification and sequencing of the ITS region and tef1, chi18-5, and $r p b 2$ gene fragments

\begin{tabular}{|c|c|c|c|c|}
\hline Locus & Primer name & Sequence (5'-3') & $\begin{array}{c}\text { Amplicon } \\
\text { length (bp) }\end{array}$ & Reference \\
\hline \multirow{2}{*}{ ITS1 and 2} & ITS4 & TCCTCCGCTTATTGATATGC & \multirow{2}{*}{650} & White et al. (1990) \\
\hline & ITS6 & GAAGGTGAAGTCGTAACAAGG & & Cooke and Duncan (1997) \\
\hline \multirow{3}{*}{ tef 1} & $\mathrm{EF} 1-728 \mathrm{~F}$ & CATCGAGAAGTTCGAGAAGG & \multirow{3}{*}{1200} & Carbone and Kohn (1999) \\
\hline & TEF-LLErev & AACTTGCAGGCAATGTGG & & Jaklitsch et al. (2006) \\
\hline & tef790 & GGGAGCGTCTGTGAATTG & & This study \\
\hline \multirow{4}{*}{ chi18-5 } & & AGCWAGCACSGATGCCAAC & \multirow{2}{*}{800} & \multirow{2}{*}{$\begin{array}{l}\text { Kullnig-Gradinger et al. } \\
(2002)\end{array}$} \\
\hline & chit42-2ar & AGGTTCTGAGTYGWGTCCA & & \\
\hline & ech1f & CACTTCACCATGTTGGGCTTCCTC & \multirow[b]{2}{*}{1200} & \multirow[b]{2}{*}{ Fekete et al. (1996) } \\
\hline & ech1r & GATCTCTAGTTGAGACCGCTTCGG & & \\
\hline \multirow{2}{*}{$r p b 2$} & RPB2-210up & TGGGGWGAYCARAARAAGG & \multirow{2}{*}{1150} & \multirow{2}{*}{ www.isth.info } \\
\hline & RPB2-1450low & CATRATGACSGAATCTTCCTGGT & & \\
\hline
\end{tabular}

a additional primer used for sequencing reactions of T. harzianum species complex strains

\section{Phylogenetic analysis}

For phylogenetic reconstruction, sequences of the tef 1 gene fragment (fourth and fifth intron, and a part of the sixth exon), generated in this study, published before by Hoyos-Carvajal et al. (2009), López-Quintero et al. (2013), Jaklitsch and Voglmayr (2015) and with NCBI GenBank taxonomic affiliation of $T$. rogersonii, T. austrokoningii, T. pararogersonii, T. subeffusum and as outgroups $T$. strigosellum, T. strigosum were used. Based on strain names or sequence accession numbers, sequences were retrieved from NCBI (www.ncbi.nlm.nih.gov/genbank) and compared using BLASTclust (toolkit.tuebingen.mpg.de/blastclust) with clustering of $100 \%$ identity sequence. A single GenBank sequence was selected for each sequence type (NCBI GenBank accessions are provided at Figure 1). Sequence alignments were prepared with ClustalX (Thompson et al. 1997) and manually edited using CLC Genomic Workbench 7.5 (CLCBio). Sequence sets of equal length were again processed with BLASTclust.
The obtained alignments were cleaned with a less stringent selection option using GBlocksServer (molevol.cmima.csic.es/castresana/Gblocks_ser ver.html). The substitution model, nucleotide frequencies and substitution values were estimated with jModelTest (Darriba et al. 2012), with the AICc selection criterion (model $\mathrm{HKY}+\mathrm{G},-\operatorname{lnL}=1730.6888, \mathrm{~K}=75$, freq $\mathrm{A}=$ 0.2004 , freqC $=0.2771$, freqG $=0.2086$, freq $\mathrm{T}=$ $0.3139, \mathrm{ti} / \mathrm{tv}=1.5292$, gamma $=0.387)$. Metropolis-coupled Markov chain Monte Carlo (MCMCMC) analysis was performed with 2 runs for 3 million generations with 4 chains with heating coefficient $\lambda=0.2$ with MrBayes 3.2.2 x64 (Ronquist et al. 2012).

\section{RESULTS}

Among the 104 Trichoderma strains collected for PGPF or BCA selection for application in agricultural and horticultural production, 100 strains were clearly identified at the species level. PCR 
amplification, sequencing, and sequence analysis of ITS 1 and 2 regions and the tef1, chi18-5, and $r p b 2$ gene fragments allowed for reliable identification of strains that belong to 15 Trichoderma species. The highest number of strains was identified as T. atroviride (39 strains), T. harzianum sensu stricto (22 strains), T. lentiforme (9 strains), T. virens (9 strains), and T. simmonsii (6 strains) (Table 1). Single strains were identified as: T. atrobrunneum
(3 strains), T. gamsii (2 strains), T. hamatum (2 strains), T. pleuroticola (2 strains), T. aggressivum f. europaeum (1 strain), T. citrinoviride (1 strain), T. crassum (1 strain), T. spirale (1 strain), $T$. tomentosum (1 strain), and $T$. viridescens (1 strain) (Table 1). Four loci-based identification of T. atroviride strains was in agreement with identification presented by Skoneczny et al. (2015).

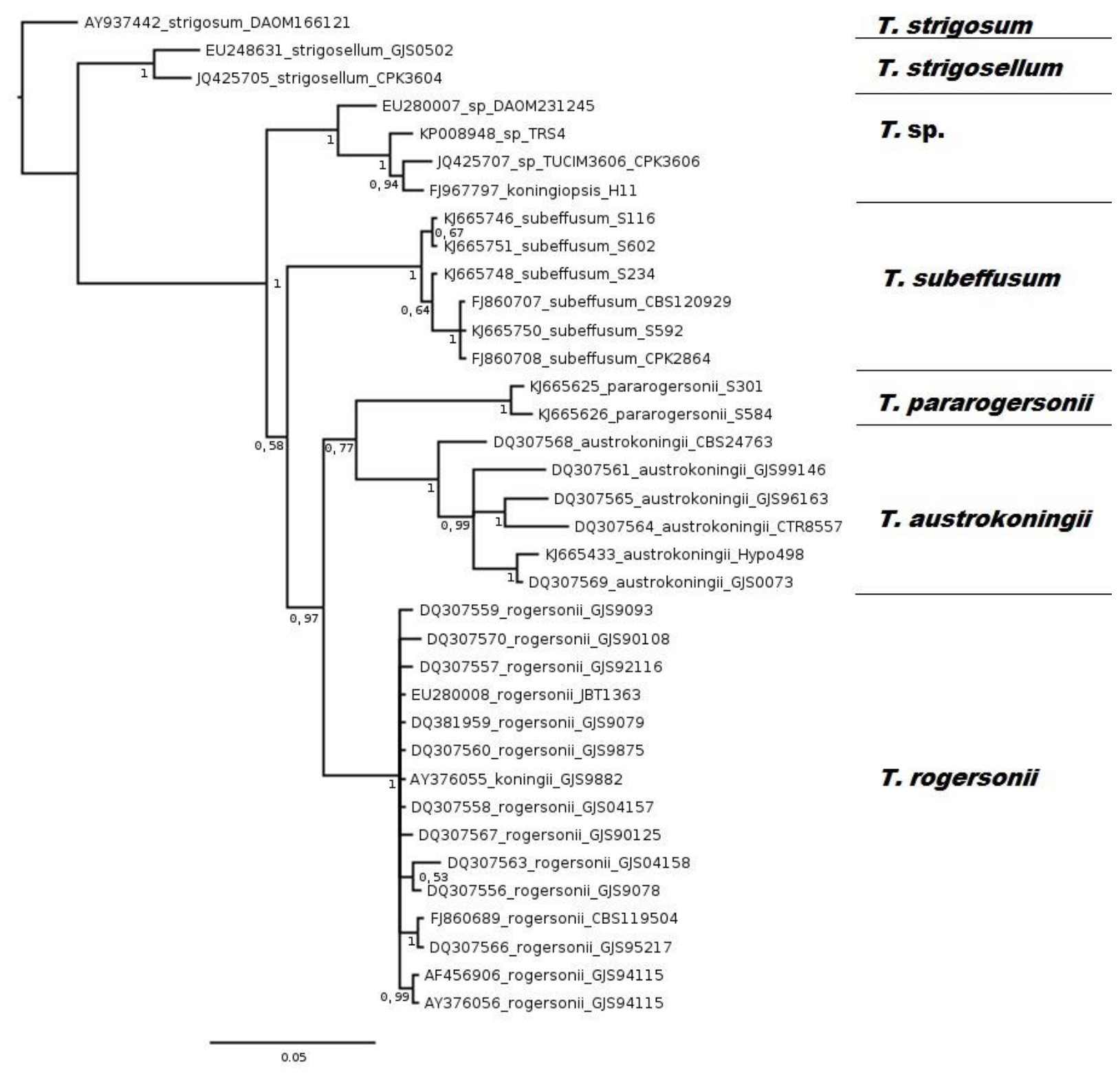

Fig. 1. Bayesian tef1 $50 \%$ majority rule tree of the Rogersonii clade of Trichoderma with T. strigosum and T. strigosellum as outgroups. Node numbers indicate Bayesian posterior probability values. The names presented here indicate NCBI GenBank accession numbers and strain names. Species are grouped and names are listed on the right. TRS4, TRS29, and TRS33 tef1 sequences are identical (NCBI GenBank Accession Numbers are: KP008948, KP008949, and KP008950) 
Species identification performed with TrichOKEY and TrichoBLAST based only on ITS1 and 2, tef 1 , and $r p b 2$ sequences was not sufficient to identify $T$. gamsii and T. viridescens. Also all strains from $T$. harzianum species complex were identified as $T$. harzianum. ITS1 and 2, and tef 1 sequences only were not sufficient to identify $T$. tomentosum and $T$. crassum. Based on tef 1 and $r p b 2$ only, identification of $T$. pleuroticola was not possible. The tef 1 sequences were insufficient to identify T. citrinoviride and T. spirale, and T. atroviride strains were not identified based on $r p b 2$. NCBI GenBank BLAST analysis of all investigated loci fulfilled identifications, however, chi18-5 sequences from T. gamsii, T. crassum, T. spirale, and T. pleuroticola in GenBank were not present. The most conclusive identification was obtained after TrichoMARK selection of 4th intron from tef 1 sequences and NCBI GenBank BLAST search.

Molecular species identification was inconclusive for four strains (TRS4, TRS29, TRS33, and TRS73). It was found that strain TRS73 belongs to the $T$. harzianum species complex ( $T$. cf. harzianum), however, the species could not be identified. Based on tef1 phylogeny, strains TRS4, TRS29, and TRS33, isolated from mushroom farms, clustered together with the previously described strains DAOM231245 and TUCIM3606 which represents separate species from $T$. rogersonii, $T$. austrokoningii, T. pararogersonii and T. subeffusum in the Rogersonii clade (Fig. 1, Table 1).

\section{DISCUSSION}

In this study we identified Trichoderma strains collected in order to select the most suitable ones for application as PGPF or BCA with organic wastes in vegetable production. The preliminary classification of these strains to T. atroviride, T. harzianum, and $T$. virens, which was based on colony morphology and microscopic observations, was insufficient to reliably determine species. Within the analysed strains, several of them appeared to have been misidentified.
We used the comparative sequence analyses of ITS1 and ITS2, tef1, chi18-5, and rpb2 loci to prepare a detailed molecular identification of strains. The main group of isolates was identified as members of three species, that is, T. atroviride (39 strains), T. harzianum sensu stricto (22 strains), and $T$. virens (9 strains). In recent decades, these species have often been recognised as the most commonly proposed candidates for biocontrol. Surprisingly, the latest studies on strains present in commercially available BCA preparations, which were previously defined as $T$. harzianum, showed that these strains were recognised as: T. afroharzianum in Canna AkTRIvator ${ }^{\circledR}$ and Trianum ${ }^{\circledR}, T$. guizhouense in Promot ${ }^{\circledR} \mathrm{WP}$, and T. simmonsii in WP Trichosan ${ }^{\circledR}$ and Vitalin ${ }^{\circledR}$. All of these species belong to the $T$. harzianum species complex, however, they are not $T$. harzianum sensu stricto (Chaverri et al. 2015). This suggests that during PGPF/BCA strain selection, more attention should be paid to the $T$. harzianum species complex than to T. harzianum sensu stricto, which is highly clonal and represents low genetic diversity (Druzhinina et al. 2010). In the strain collection analysed in this study, the $T$. harzianum species complex was represented by $T$. atrobrunneum (3 strains), T. lentiforme (9 strains), T. simmonsii (6 strains) and one strain TRS73, which possibly represents an unknown lineage (Table 1).

Within the strains used in this study, three strains belonging to T. aggressivum (TRS27) and T. pleuroticola (TRS70 and TRS120) were detected. These are morphologically similar to T. harzianum, however, they are pathogenic to cultivated mushrooms. Therefore, these strains will be excluded from further studies aimed at PGPF/BCA strain selection for vegetable cultivation. On the other hand, Komoń-Żelazowska et al. (2007) mentioned that two strains of T. pleuroticola were selected as biofungicides against soilborne plant diseases. Such fungal agents can be potentially risky for use in vegetable cultivation because they represent a potential source of mushroom pathogens. Our study confirms the necessity of careful molecular species identification 
of microorganisms used for agricultural and horticultural applications.

Recently, Jaklitsch and Voglmayr (2015) recognised a new species, $T$. pararogersonii, in the Rogersonii clade that includes $T$. rogersonii, T. austrokoningii and $T$. subeffusum. Our study suggests that the Rogersonii clade comprises one more undescribed species represented by three Trichoderma strains, that is, TRS4, TRS29, and TRS33, (this study) and strains isolated from Central America DAOM 231245 and C.P.K. 3606. It has been proposed that DAOM 231245 and C.P.K. 3606 represent a putative new Trichoderma species described as T. sp. 435 (Hoyos-Carvajal et al. 2009) and T. sp. C.P.K. 3606 (López-Quintero et al. 2013). The phylogeny reconstruction that was performed in this study supports this observation (Fig. 1).

Our study confirmed the usefulness of multilocus-based molecular identification of Trichoderma species. Such an identification, based on 4 loci (ITS1 and 2, tef1, chi18-5, and rpb2), was successful for 100 out of the 104 (96\%) Trichoderma strains that were analyzed in this study. Based on such species identification, a selection of strains that could be potentially useful as PGPF or BCA in vegetable production could be improved and better focused. Strains belonging to Trichoderma species pathogenic to cultivated mushrooms can be eliminated from such programs. Also, strains belonging to taxonomic groups characterised by biocontrol properties can be distinguished, for example, in strains belonging to the T. harzianum species complex. The study also showed gaps in Trichoderma taxonomy and the existence of new, unestablished species. For even more successful Trichoderma species identification, further adjustment of Trichoderma taxonomy is necessary.

\section{Acknowledgements}

We are grateful to Dr. Irina Druzhinina (TUV, Vienna, Austria) for help in the better understanding of Trichoderma identification and phylogeny.

This work was conducted as a part of the project "Polish Trichoderma strains in plant protection and organic waste management" Priority 1.3.1, subject area "Bio", co-financed by The European Union through the European Regional Development Fund within the Innovative Economy Operational Program, 2007-2013, under project No [UDA-POIG.01.03.01-00-129/09-08].

\section{REFERENCES}

Aldrich J., Cullis C.A. 1993. RAPD analysis in flax: optimization of yield and reproducibility using KlenTaq 1 DNA polymerase, Chelex 100, and gel purification of genomic DNA. Plant Molecular Biology Reporter 11: 128-141. DOI: 10.1007/BF02670471.

Altschul S.F., Madden T.L., Schäffer A.A., Zhang J., Zhang Z., Miller W., Lipman D.J. 1997. Gapped BLAST and PSI-BLAST: a new generation of protein database search programs. Nucleic Acids Research 25: 3389-3402. DOI: $10.1093 / \mathrm{nar} / 25.17 .3389$.

Atanasova L., Druzhinina I.S., Jaklitsch W.M. 2013. Two hundred Trichoderma species recognized on the basis of molecular phylogeny. In: Mukherjee P.K., Horwitz B.A., Singh U.S., Mukherjee M., Schmoll M. (Eds.), Trichoderma: biology and applications. CABI, Wallingford, UK, pp. 10-42. DOI: 10.1079/9781780642475.0010.

Benítez T., Rincón A.M., Limón M.C., Codón A.C. 2004. Biocontrol mechanisms of Trichoderma strains. International Microbiology 7: 249-260.

Błaszczyk L., Popiel D., Chełkowski J., Koczyk G., Samuels G.J., Sobieralski K., Siwulski M. 2011. Species diversity of Trichoderma in Poland. Journal of Applied Genetics 52: 233-243. DOI: 10.1007/s13353-011-0039-z.

Carbone I., Kohn L.M. 1999. A method for designing primer sets for speciation studies in filamentous ascomycetes. Mycologia 91: 553-556. DOI: 10.2307/3761358

Chaverri P., Branco-Rocha F., Jaklitsch W.M., Gazis R.O., Degenkolb T., Samuels G.J. 2015. Systematics of the Trichoderma harzianum species complex and the re-identification of commercial biocontrol strains. Mycologia 107: 558-590. DOI: 10.3852/14-147.

Chaverri P., Castlebury L.A., Samuels G.J., Geiser D.M. 2003. Multilocus phylogenetic structure within the Trichoderma harzianum/Hypocrea lixii complex. Molecular Phylogenetics and Evolution 27: 302313. DOI: 10.1016/S1055-7903(02)00400-1. 
Chaverri P., Gazis R.O., Samuels G.J. 2011. Trichoderma amazonicum, a new endophytic species on Heveabrasiliensis and H. guianensis from the Amazon basin. Mycologia 103: 139-151. DOI: 10.3852/10-078.

Cooke D.E.L., Duncan J.M. 1997. Phylogenetic analysis of Phytophthora species based on ITS1 and ITS2 sequences of the ribosomal RNA gene repeat. Mycological Research 101: 667-677. DOI: 10.1017/S0953756296003218.

Darriba D., Taboada G.L., Doallo R., Posada D. 2012. jModelTest 2: more models, new heuristics and parallel computing. Nature Methods 9: 772. DOI: 10.1038/nmeth.2109.

Dodd S.L., Lieckfeldt E., Samuels G.J. 2003. Hypocrea atroviridis sp. nov., the teleomorph of Trichoderma atroviride. Mycologia 95: 27-40. DOI: $10.2307 / 3761959$.

Druzhinina I.S., Kopchinskiy A.G., Komoń M., Bissett J., Szakacs G., Kubicek C.P. 2005. An oligonucleotide barcode for species identification in Trichoderma and Hypocrea. Fungal Genetics and Biology 42: 813-828. DOI: 10.1016/j.fgb.2005.06.007.

Druzhinina I.S., Kubicek C.P., Komoń-Żelazowska M., Mulaw T.B., Bissett J. 2010. The Trichoderma harzianum demon: complex speciation history resulting in coexistence of hypothetical biological species, recent agamospecies and numerous relict lineages. BMC Evolutionary Biology 10: 94. DOI: 10.1186/1471-2148-10-94.

Fekete C., Weszely T., Hornok L. 1996. Assignment of a PCR-amplified chitinase sequence cloned from Trichodermahamatum to resolved chromosomes of potential biocontrol species of Trichoderma. FEMS Microbiology Letters 145: 385-391. DOI: 10.1111/j.1574-6968.1996.tb08605.x.

Gal-Hemed I., Atanasova L., Komoń-Żelazowska M., Druzhinina I.S., Viterbo A., Yarden O. 2011. Marine isolates of Trichoderma spp. as potential halotolerant agents of biological control for arid-zone agriculture. Applied and Environmental Microbiology 77: 5100-5109. DOI: 10.1128/AEM.00541-11.

Gazis R., Chaverri P. 2010. Diversity of fungal endophytes in leaves and stems of wild rubber trees $(\mathrm{He}$ veabrasiliensis) in Peru. Fungal Ecology 3: 240254. DOI: 10.1016/j.funeco.2009.12.001.
Harman G.E. 2006. Overview of mechanisms and uses of Trichoderma spp. Phytopathology 96: 190-194. DOI: 10.1094/PHYTO-96-0190.

Harman G.E., Howell C.R., Viterbo A., Chet I., Lorito M. 2004. Trichoderma species - opportunistic, avirulent plant symbionts. Nature Reviews Microbiology 2: 43-56. DOI: 10.1038/nrmicro797.

Hermosa M.R., Grondona I., Iturriaga E.A., DiazMinguez J.M., Castro C., Monte E., Garcia-Acha I. 2000. Molecular characterization and identification of biocontrol isolates of Trichoderma spp. Applied and Environmental Microbiology 66: 1890-1898. DOI: 10.1128/AEM.66.5.1890-1898.2000.

Hermosa R., Rubio M.B., Cardoza R.E., Nicolás C., Monte E., Gutiérrez S. 2013. The contribution of Trichoderma to balancing the costs of plant growth and defense. International Microbiology 16: 69-80. DOI: $10.2436 / 20.1501 .01 .181$.

Hoyos-Carvajal L., Orduz S., Bissett J. 2009. Genetic and metabolic biodiversity of Trichoderma from Colombia and adjacent neotropic regions. Fungal Genetics and Biology 46: 615-631. DOI: 10.1016/j.fgb.2009.04.006.

Jaklitsch W.M. 2011. European species of Hypocrea part II: species with hyaline ascospores. Fungal Diversity 48: 1-250. DOI: 10.1007/s13225-011-0088-y.

Jaklitsch W.M., Samuels G.J., Dodd S.L., Lu B.S., Druzhinina I.S. 2006. Hypocrea rufa/Trichoderma viride: a reassessment, and description of five closely related species with and without warted conidia. Studies in Mycology 56: 135-177. DOI: 10.3114/sim.2006.56.04.

Jaklitsch W.M., Samuels G.J., Ismaiel A., Voglmayr H. 2013. Disentangling the Trichoderma viridescens complex. Persoonia 31: 112-146. DOI: 10.3767/003158513X672234.

Jaklitsch W.M., Voglmayr H. 2015. Biodiversity of Trichoderma (Hypocreaceae) in Southern Europe and Macaronesia. Studies in Mycology 80: 1-87. DOI: 10.1016/j.simyco.2014.11.001.

Kancelista A., Tril U., Stempniewicz R., Piegza M., Szczech M., Witkowska D. 2013. Application of lignocellulosic waste materials for the production and stabilization of Trichoderma biomass. Polish Journal of Environmental Studies 22: 1083-1090.

Kindermann J., El-Ayouti Y., Samuels G.J., Kubicek C.P. 1998. Phylogeny of the genus Trichoderma based on 
sequence analysis of the internal transcribed spacer region 1 of the rDNA cluster. Fungal Genetics and Biology 24: 298-309. DOI: 10.1006/fgbi.1998.1049.

Komoń-Żelazowska M., Bissett J., Zafari D., Hatvani L., Manczinger L., Woo S., Lorito M., Kredics L., Kubicek C.P., Druzhinina I.S. 2007. Genetically closely related but phenotypically divergent Trichoderma species cause green mold disease in oyster mushroom farms worldwide. Applied and Environmental Microbiology 73: 7415-7426. DOI: 10.1128/AEM.01059-07.

Kopchinskiy A., Komoń M., Kubicek C.P., Druzhinina I.S. 2005. TrichoBLAST: A multilocus database for Trichoderma and Hypocrea identifications. Mycological Research 109: 658-660. DOI: 10.1017/S0953756205233397.

Kredics L., Hatvani L., Naeimi S., Körmöczi P., Manczinger L., Vágvölgyi C., Druzhinina I. 2014. Biodiversity of the genus Hypocrea/Trichoderma in different habitats. In: Gupta V.K., Schmoll M., Herrera-Estrella A., Upadhyay R.S., Druzhinina I., Tuohy M.G. (Eds.), Biotechnology and biology of Trichoderma. Elsevier, Amsterdam, pp. 3-24. DOI: 10.1016/B978-0-444-59576-8.00001-1.

Kullnig C.M., Krupica T., Woo S.L., Mach R.L., Rey M., Benítez T., Lorito M., Kubicek C.P. 2001. Confusion abounds over identities of Trichoderma biocontrol isolates. Mycological Research 105: 769772. DOI: 10.1017/S0953756201229967.

Kullnig-Gradinger C.M., Szakacs G., Kubicek C.P. 2002. Phylogeny and evolution of the genus Trichoderma: a multigene approach. Mycological Research 106: 757-767. DOI: 10.1017/S0953756202006172.

Longa C.M.O., Savazzini F., Tosi S., Elad Y., Pertot I. 2009. Evaluating the survival and environmental fate of the biocontrol agent Trichoderma atroviride $\mathrm{SC} 1$ in vineyards in northern Italy. Journal of Applied Microbiology 106: 1549-1557. DOI: 10.1111/j.1365-2672.2008.04117.x.

López-Quintero C.A., Atanasova L., Franco-Molano A.E., Gams W., Komoń-Żelazowska M., Theelen B., Müller W.H., Boekhout T., Druzhinina I. 2013. DNA barcoding survey of Trichoderma diversity in soil and litter of the Colombian lowland
Amazonian rainforest reveals Trichoderma strigosellum sp. nov. and other species. Antonie van Leeuwenhoek, Journal of Microbiology 104: 657 674. DOI: 10.1007/s10482-013-9975-4.

Monfil V.O., Casas-Flores S. 2014. Molecular mechanisms of biocontrol in Trichoderma spp. and their applications in agriculture. In: Gupta V.K., Schmoll M., Herrera-Estrella A., Upadhyay R.S., Druzhinina I., Tuohy M.G. (Eds.), Biotechnology and biology of Trichoderma. Elsevier, Amsterdam, pp. 429- 453. DOI: 10.1016/B978-0-444-59576-8.00032-1.

Mulaw T.B., Kubicek C.P., Druzhinina I.S. 2010. The rhizosphere of Coffea arabica in its native highland forests of Ethiopia provides a niche for a distinguished diversity of Trichoderma. Diversity 2: 527 549. DOI: 10.3390/d2040527.

Ronquist F., Teslenko M., van der Mark P., Ayres D.L., Darling A., Höhna S., et al. 2012. MrBayes 3.2: efficient Bayesian phylogenetic inference and model choice across a large model space. Systematic Biology 61: 539-542. DOI: 10.1093/sysbio/sys029.

Samuels G.J., Dodd S.L., Lu B.S., Petrini O., Schroers H.J., Druzhinina I.S. 2006. The Trichoderma koningii aggregate species. Studies in Mycology 56: 67-133. DOI: 10.3114/sim.2006.56.03.

Skoneczny D., Oskiera M., Szczech M., Bartoszewski G. 2015. Genetic diversity of Trichoderma atroviride strains collected in Poland and identification of loci useful in detection of within-species diversity. Folia Microbiologica 60: 297-307. DOI: 10.1007/s12223-015-0385-z.

Smolińska U., Gołębiewska E., Kowalska B., Kowalczyk W., Szczech M. 2014a. Materiały odpadowe jako nośniki antagonistycznych grzybów Trichoderma. Inżynieria i Ochrona Środowiska 17: 5-20.

Smolińska U., Kowalska B., Kowalczyk W., Szczech M. 2014b. The use of agro-industrial wastes as carriers of Trichoderma fungi in the parsley cultivation. Scientia Horticulturae 179: 1-8. DOI: 10.1016/j.scienta.2014.08.023.

Stewart A., Hill R. 2014. Applications of Trichoderma in plant growth promotion. In: Gupta V.K., Schmoll M., Herrera-Estrella A., Upadhyay R.S., Druzhinina I., Tuohy M.G. (Eds.), Biotechnology and biology of Trichoderma. Elsevier, Amsterdam, pp. 415-428. DOI: 10.1016/b978-0-444-595768.00031-x. 
Thompson J.D., Gibson T.J., Plewniak F., Jeanmougin F., Higgins D.G. 1997. The CLUSTAL_X windows interface: flexible strategies for multiple sequence alignment aided by quality analysis tools. Nucleic Acids Research 25: 4876-4882. DOI: 10.1093/nar/25.24.4876.

Vandroemme J., Baeyen S., Van Vaerenbergh J., De Vos P., Maes M. 2008. Sensitive real-time PCR detection of Xanthomonas fragariae in strawberry plants. Plant Pathology 57: 438-444. DOI: 10.1111/j.1365-3059.2007.01813.x.

White T.J., Bruns T., Lee S., Taylor J. 1990. Amplification and direct sequencing of fungal ribosomal RNA genes for phylogenetics. In: Innis M.A., Gelfand D.H., Sninsky J.J., White T.J. (Eds.), PCR protocols: a guide to methods and applications. Academic Press, San Diego, CA, pp. 315-322. DOI: 10.1016/b978-0-12-372180-8.50042-1. 